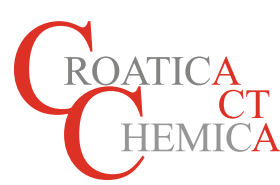

\title{
Order-Disorder in Diaquobis(salicylato)copper(II) Revisited
}

\author{
Martin Lutz, Loes M. J. Kroon-Batenburg*
}

\author{
Crystal and Structural Chemistry, Bijvoet Center for Biomolecular Research, Utrecht University, Padualaan 8, 3584 CH Utrecht, The Netherlands \\ * Corresponding author's e-mail address: I.m.j.kroon-batenburg@uu.nl
}

RECEIVED: May 4, 2018 * REVISED: June 21, 2018 * ACCEPTED: June 28, 2018

THIS PAPER IS DEDICATED TO DR. BISERKA KOJIĆ-PRODIĆ ON THE OCCASION OF HER 80Th BIRTHDAY

\begin{abstract}
We re-investigated the crystal structure of diaquobis(salicylato)copper(II), CuSal, which is an OD material with disordered layer stacking. The diffraction pattern consists of sharp Bragg spots and diffuse streaks.

From the Bragg reflections we determined the ordered part of the structure, which can either be described by an orthorhombic or a twinned monoclinic lattice. The main motif of the structure consists of one-dimensional coordination polymers that are connected by hydrogen bonds into two-dimensional layers.

The layer stacking is disordered and from simulations with a Markovian growth models we could fully explain the diffuse streaks of the current structure when the layers are stacked randomly.
\end{abstract}

Keywords: diffuse scattering, OD structure, hydrogen-bonded layers, copper complex.

\section{INTRODUCTION}

$\mathbf{T}$ HE disorder in the crystal structure of the title compound diaquobis(salicylato)copper(II) (CuSal) has been recognized first by Dornberger-Schiff \& Malinowski.[1] In a series of publications this structure has later been investigated in detail by Jagner et al. on the basis of the Order-Disorder Theory (OD theory). ${ }^{[2-5]}$ The diffraction pattern is characterized by sharp Bragg reflections for $h=$ even and diffuse streaks for $h=o d d$. The diffuse streaks are running in the $c^{*}$ direction. According to Jagner et al.,[2] the sharp reflections can be indexed either by an orthorhombic or a monoclinic unit cell. These correspond to the phases $\mathrm{MDO}_{1}$ (orthorhombic) and $\mathrm{MDO}_{2}$ (monoclinic), where MDO stands for Maximum Degree of Order. In terms of the OD theory, the sharp Bragg reflections can be named family reflections. On the locations of the diffuse streaks, the orthorhombic and monoclinic reciprocal unit cells are non-overlapping. Jagner et al. ${ }^{[2]}$ find explicit intensity maxima on the streaks which would correspond to either of them.

The structure description of Jagner et al. ${ }^{[2]}$ is characterized by one-dimensional coordination polymers along the $\mathbf{b}$ axis, which are present in both the orthorhombic and the monoclinic description. Hydrogen atoms were not determined and their influence on the crystal packing not discussed. In the monoanionic salicylate ligand it is unclear whether the carboxylate or the hydroxyl group remain protonated. In addition there is an ambiguity for the position of the hydroxy group. Jagner et al. used a disorder model which has a partial occupancy for the hydroxy group on both ortho positions.

By the reaction of sodium salicylate with copper(II) sulfate we obtained the polymeric $\left[\mathrm{Cu}_{2}\left(\mathrm{C}_{7} \mathrm{H}_{4} \mathrm{O}_{3}\right)_{2}\left(\mathrm{H}_{2} \mathrm{O}\right)_{2}\right]$. $2 \mathrm{H}_{2} \mathrm{O}$ that contains the dianionic salicylate ligand $\mathrm{Sal}^{2-}$ and which we published earlier. ${ }^{[6]}$ In the same crystallization batch we also obtained CuSal of the current study which contains the monoanionic ligand HSal-. The two crystal forms can easily be distinguished by their colour. Crystals with the dianionic salicylate are brown, ${ }^{[6]}$ while the crystals of the current study with monoanionic salicylate are green.

In the context of our studies on salicylates we redetermined the structure of CuSal. With the new diffraction data, hydrogen atoms could be determined and the hydrogen bonding scheme can be discussed. While OD theory is an established, thorough and complete theory of 
(hOl)

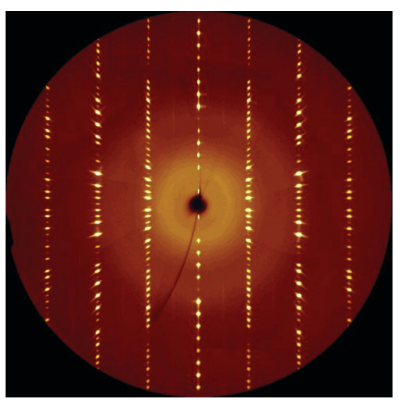

(hOl)

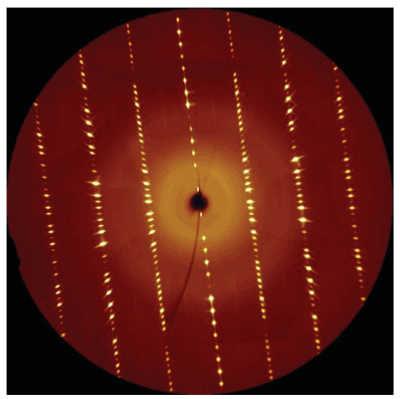

(h1l)

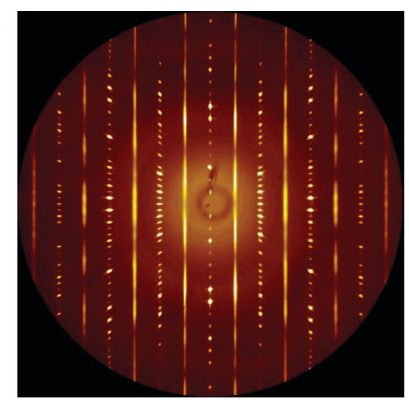

(h1l)

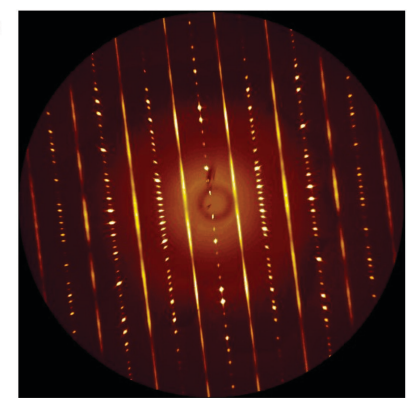

$(1 \mathrm{kl})$

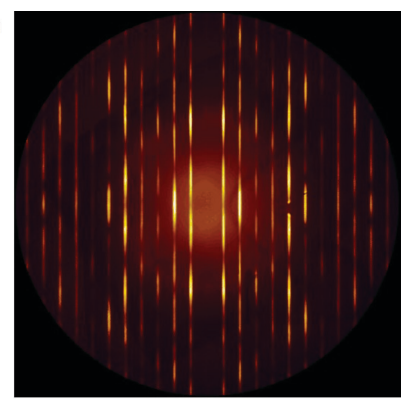

$(1 \mathrm{kl})$

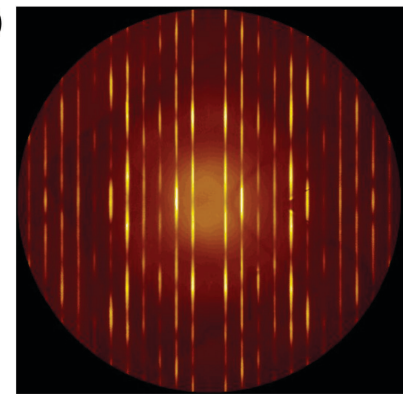

Figure 1. Slices through reciprocal space reconstruction of the data. An orthorhombic (top) and monoclinic (bottom) unit cell were used for their generation with the program precession in the Eval15 suite. ${ }^{[10]}$ Diffuse streaks are running in the corresponding $\mathrm{c}^{*}$ directions and are absent in the zeroth $k$ layers.

symmetry ${ }^{[7-9]}$, the symmetry theory does not explain the intensity distribution in the diffraction pattern. We therefore discuss explanations for the diffuse scattering intensities.

\section{RESULTS AND DISCUSSION}

\section{Molecular and Crystal Structure}

Figure 1 shows two-dimensional reconstructions of the diffraction pattern. The main features are consistent with the observations of Jagner et al. ${ }^{[2]}$ and Falch et al. ${ }^{[11]}$ The major difference is in the diffuse streaks. The streaks of the current pattern have no explicit intensity maxima whereas Jagner et al. could assign maxima to either the orthorhombic or monoclinic unit cell. Consequently, for the refinement of the unit cells and for the evaluation of the $X$ ray intensities only reflections with $h=$ even could be used in our case. The data completeness is thus halved. We can choose both lattices for indexing. More experimental details are given below in Table 3 and Table 4. The transformation matrix for the $h=$ even layers is provided in Equation (1). Slight deviations in the cell parameters of both settings are due to independent data integrations and independent unit cell refinements.

$$
\left(\begin{array}{l}
h_{\text {mono }} \\
k_{\text {mono }} \\
l_{\text {mono }}
\end{array}\right)=\left(\begin{array}{ccc}
1 & 0 & 0 \\
0 & 1 & 0 \\
-0.5 & 0 & 1
\end{array}\right)\left(\begin{array}{l}
h_{\text {ortho }} \\
k_{\text {ortho }} \\
l_{\text {ortho }}
\end{array}\right)
$$

Despite the presence of diffuse streaks we set out to determine the atomic structure. Especially interesting is an analysis of the hydrogen bonding situation which has not been discussed by Jagner et al. ${ }^{[2]}$

\section{Orthorhombic Phase $\mathbf{M D O}_{1}$}

Reflections with $h=$ even can alternatively be indexed with an orthorhombic or monoclinic lattice. In the orthorhombic setting, the asymmetric unit contains two copper atoms, four monoanionic salicylate ligands and four neutral water molecules. In contrast to the literature structure, ${ }^{[2]}$ there is no statistical disorder in the salicylate molecules present. Figure 2 shows a plot of the asymmetric unit. Two of the

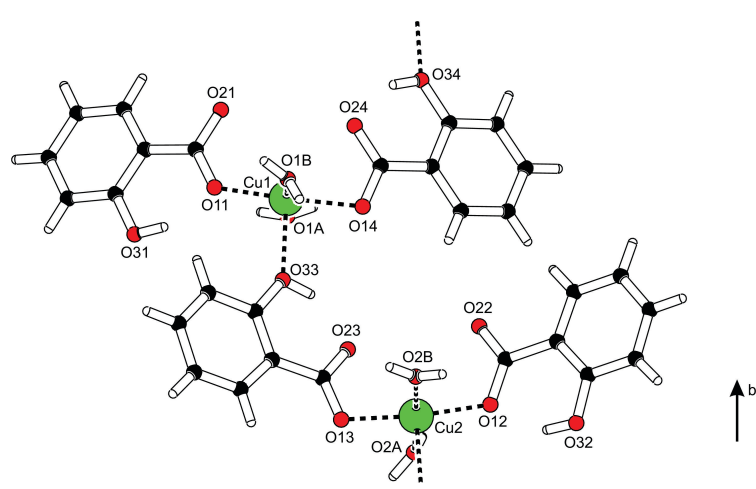

Figure 2. Asymmetric unit of the orthorhombic phase. View approximately along the $a$ axis. 
Table 1. Hydrogen bonding geometry in the orthorhombic form

\begin{tabular}{|c|c|c|c|c|}
\hline$D-H \cdots A^{(a)}$ & $\mathrm{D}-\mathrm{H} / \AA$ & $H \cdots A / \AA$ & $D \cdots A / \AA$ & $D-H \cdots A /{ }^{\circ}$ \\
\hline $\mathrm{O} 1 A-\mathrm{H} 1 A \cdots \mathrm{O} 24^{\mathrm{i}}$ & 0.85 & 1.91 & $2.706(10)$ & 155 \\
\hline $\mathrm{O} 1 A-\mathrm{H} 1 B \cdots \mathrm{O} 21^{i}$ & 0.85 & 1.89 & $2.676(13)$ & 152 \\
\hline $\mathrm{O} 1 B-\mathrm{H} 1 \mathrm{C} \cdots \mathrm{O} 13^{\mathrm{ii}}$ & 0.84 & 1.94 & $2.766(9)$ & 166 \\
\hline $\mathrm{O} 1 B-\mathrm{H} 1 D \cdots \mathrm{O} 21^{\mathrm{iii}}$ & 0.85 & 1.92 & $2.766(13)$ & 170 \\
\hline $\mathrm{O} 2 \mathrm{~A}-\mathrm{H} 2 \mathrm{~A} \cdots \mathrm{O} 14^{\mathrm{iv}}$ & 0.86 & 1.95 & $2.762(10)$ & 158 \\
\hline $\mathrm{O} 2 A-\mathrm{H} 2 B \cdots \mathrm{O} 22^{\mathrm{iv}}$ & 0.85 & 2.05 & $2.713(12)$ & 135 \\
\hline $\mathrm{O} 2 \mathrm{~B}-\mathrm{H} 2 \mathrm{C} \cdots \mathrm{O} 23^{\mathrm{ii}}$ & 0.85 & 1.85 & $2.634(11)$ & 152 \\
\hline $\mathrm{O} 2 B-\mathrm{H} 2 D \cdots O 22^{i i}$ & 0.85 & 1.88 & $2.620(12)$ & 145 \\
\hline $\mathrm{O} 31-\mathrm{H} 31 \cdots \mathrm{O} 11$ & 0.84 & 1.72 & $2.493(12)$ & 153 \\
\hline $\mathrm{O} 32-\mathrm{H} 32 \cdots \mathrm{O} 12$ & 0.84 & 1.92 & $2.653(9)$ & 145 \\
\hline $\mathrm{O} 33-\mathrm{H} 33 \cdots \mathrm{O} 23$ & 0.84 & 1.77 & $2.513(8)$ & 147 \\
\hline $\mathrm{O} 34-\mathrm{H} 34 \cdots \mathrm{O} 24$ & 0.84 & 1.74 & $2.494(8)$ & 148 \\
\hline
\end{tabular}

(a) Symmetry codes: (i) $x-0.5,1-y, z$; (ii) $x+0.5,-y, z$; (iii) $x+0.5,1-y, z$; (iv) $x-0.5,-y, z$.

monoanionic salicylate ligands are bridging the copper centers which leads to a one-dimensional polymeric chain in $\mathbf{b}$ direction. The other two salicylate ligands and the water molecules are non-bridging. All crystallographically independent atoms belong to the same chain.

Monoanionic salicylate complexes are quite common. In the majority of cases, the carboxylate group is deprotonated and the hydroxyl group is protonated. ${ }^{[12]}$ This situation is also found here for all four independent salicylate ligands. The hydroxyl groups are only involved in intramolecular hydrogen bonds with the carboxylate oxygen of the same salicylate as acceptor. In the terminal salicylates, the metal-coordinated oxygen is the acceptor, and in the bridging salicylates it is the non-coordinated oxygen. The water molecules act as donors for intermolecular hydrogen bonds with again the carboxylate oxygens as acceptors. These hydrogen bonds connect the one-dimensional coordination chains into a two-dimensional network parallel to the ab plane. An overview of the hydrogen bonded geometries is provided in Table 1 . The two-dimensional layers are further stabilized by $\pi \cdots \pi$ interactions between the phenyl rings of the salicylate ligands within the layers. Ring $\cdots$ ring distances were determined as $3.423(2)-3.508(3) \AA$.

In accordance with the description of, ${ }^{[2]}$ the environment of both $\mathrm{Cu}$ centers is five-coordinated with a square pyramidal geometry as indicated by the $\tau$ parameters $^{[13]}$ of 0.10 and 0.06 for Cu1 and Cu2, respectively. It should be noted that both $\mathrm{Cu}$ centers have a sixth oxygen in proximity which originates from the carboxylate group of the bridging salicylate. The corresponding distances

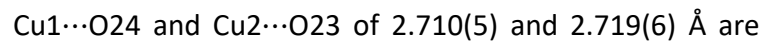

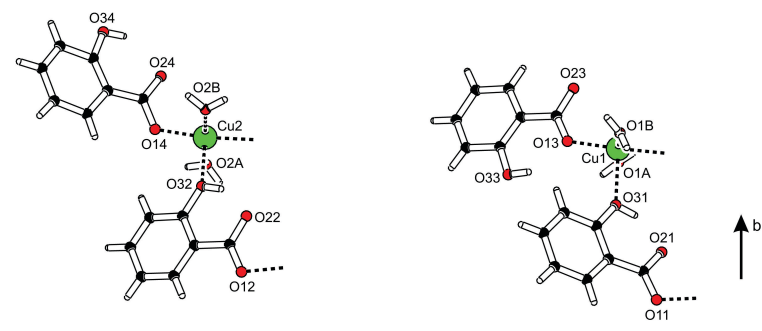

Figure 3. Asymmetric unit of the monoclinic phase. View approximately along $-a$.

quite long. Such long bonds have been observed in octahedral, Jahn-Teller distorted $\mathrm{Cu}(\mathrm{II})$ complexes, but the corresponding $\mathrm{O}-\mathrm{Cu} \cdots \mathrm{O}$ angles of 53.5(2) and 53.16(18) ${ }^{\circ}$ contradict an octahedral environment and a bonding situation, here. Generally, the coordination mode of asymmetric bonded carboxylate groups is difficult to determine.[14]

The orthorhombic phase has the polar, non-centrosymmetric space group $\mathrm{Pca}_{1}$. Refinement as an inversion twin results in a Flack parameter ${ }^{[15]}$ of $x=0.50(4)$ which indicates perfect twinning or centrosymmetry. This value is based on the reflections with $h=$ even only.

\section{Monoclinic Phase $\mathrm{MDO}_{2}$}

An alternative indexing of reflections with $h=$ even is possible with a monoclinic lattice. We found that quality improved if the intensities are "de-twinned" from the orthorhombic contribution (see Experimental Section).

The description in monoclinic symmetry has space group $P 2_{1} / a$. This space group has the same number of symmetry operations as the orthorhombic Pca2 ${ }_{1}$. Consequently, the number of atoms in the asymmetric unit is the same. While in the orthorhombic phase all atoms in the 
Table 2. Hydrogen bonding geometry in the monoclinic form

\begin{tabular}{|c|c|c|c|c|}
\hline $\mathrm{D}-\mathrm{H} \cdots A^{(\mathrm{a})}$ & $D-H / \AA$ & $H \cdots A / \AA$ & $D \cdots A / \AA$ & $\mathrm{D}-\mathrm{H} \cdots \mathrm{A} /{ }^{\circ}$ \\
\hline $\mathrm{O} 1 A-\mathrm{H} 1 A \cdots \mathrm{O} 21^{i}$ & 0.82 & 1.89 & $2.637(7)$ & 150 \\
\hline $\mathrm{O} 1 A-\mathrm{H} 1 B \cdots \mathrm{O} 23^{\mathrm{ii}}$ & 0.86 & 1.90 & $2.636(8)$ & 143 \\
\hline $\mathrm{O} 1 B-\mathrm{H} 1 C \cdots \mathrm{O} 11^{\mathrm{iii}}$ & 0.84 & 1.98 & $2.789(6)$ & 160 \\
\hline $\mathrm{O} 1 B-\mathrm{H} 1 D \cdots \mathrm{O} 23^{\mathrm{iv}}$ & 0.85 & 1.92 & $2.758(8)$ & 172 \\
\hline $\mathrm{O} 2 A-\mathrm{H} 2 A \cdots \mathrm{O} 12^{v}$ & 0.86 & 1.91 & $2.755(7)$ & 165 \\
\hline $\mathrm{O} 2 A-\mathrm{H} 2 B \cdots \mathrm{O} 24^{\mathrm{ii}}$ & 0.85 & 1.94 & $2.735(9)$ & 154 \\
\hline $\mathrm{O} 2 \mathrm{~B}-\mathrm{H} 2 \mathrm{C} \cdots \mathrm{O} 24^{\mathrm{iv}}$ & 0.88 & 1.87 & $2.681(9)$ & 153 \\
\hline $\mathrm{O} 2 B-\mathrm{H} 2 \mathrm{D} \cdots \mathrm{O} 22^{\mathrm{vi}}$ & 0.86 & 1.89 & $2.716(8)$ & 159 \\
\hline $\mathrm{O} 31-\mathrm{H} 31 \cdots \mathrm{O} 21$ & 0.84 & 1.76 & $2.514(5)$ & 149 \\
\hline $\mathrm{O} 32-\mathrm{H} 32 \cdots \mathrm{O} 22$ & 0.84 & 1.73 & $2.476(6)$ & 147 \\
\hline $\mathrm{O} 33-\mathrm{H} 33 \cdots \mathrm{O} 13$ & 0.84 & 1.91 & $2.649(7)$ & 145 \\
\hline $\mathrm{O} 34-\mathrm{H} 34 \cdots \mathrm{O} 24$ & 0.84 & 2.11 & $2.780(8)$ & 136 \\
\hline
\end{tabular}

(a) Symmetry codes: (i) $x, y,-z$; (ii) $x-0.5,0.5-y, z$; (iii) $x+0.5,-y-0.5, z$; (iv) $x+0.5,0.5-y, z$; (v) $x-0.5,-y-0.5, z$; (vi) $2-x,-y, 1-z$.

asymmetric unit belong to the same coordination chain, in the monoclinic phase they are in two independent chains (Figure 3). Symmetry operations of $P c a 2_{1}$ have become pseudo-operations in $P 2_{1} / a$, and vice versa. Again, the hydrogen bonding scheme leads to two-dimensional sheets in the ab plane. The hydrogen bonding geometries can be found in Table 2.

The monoclinic lattice can be transformed into a Ccentered orthorhombic lattice with doubled volume using the LEPAGE algorithm of the PLATON software. ${ }^{[16]}$ If structure factors are calculated from the monoclinic singlecrystal model, the $F_{\text {calc }}^{2}$ have $R_{\text {int }}=35 \%$ after transformation to orthorhombic C (NEWSYM routine in PLATON). The orthorhombic symmetry is thus not supported by the atomic model. Still, this orthorhombic geometry needs to be considered as a driving force for possible twinning. In the refinement we included a twin matrix corresponding to a twofold rotation about $h k l=(0,0,1)$. This leads to a complete overlap of all reflections (pseudo-orthorhombic

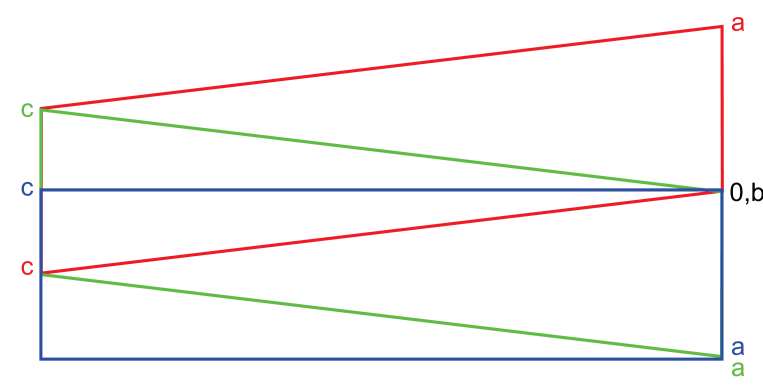

Figure 4. Orientation of the two twinned monoclinic unit cells (red and green) with respect to the orthorhombic unit cell (blue). View along the orthorhombic $b$ axis. twinning) and the twin fraction refined to 0.501(6). The relation between the different unit cells is displayed in Figure 4.

The monoclinic unit lattices overlap perfectly with each other. They overlap with the orthorhombic lattice in every second layer. The supercell common to all three unit cells has thus an orthorhombic geometry with the $c$ axis doubled. This common supercell is responsible for the sharp Bragg reflections (family reflections) in the diffraction pattern.

\section{Structure Comparison}

Both the orthorhombic and the monoclinic description of the CuSal structure are characterized by two-dimensional layers in the $a b$ plane. These layers are constructed by a coordination chain along $\mathbf{b}$ which are linked by intermolecular hydrogen bonds and $\pi \cdots \pi$ interactions into twodimensional layers. A projection along the a axis is shown in Figure 5. In this perspective, the two packing arrangements are very similar. The major difference is in the symmetry operations which are indicated by colour schemes in the drawings. In the orthorhombic description all atoms of the asymmetric unit belong to the same coordination chain, while in the monoclinic description they are divided over two symmetry-independent chains.

The stacking difference between the orthorhombic and monoclinic form can be seen in projections along the $\mathbf{b}$ axis (Figure 6) and the along the $\mathbf{c}$ axis (Figure 7). The latter is a projection perpendicular to the hydrogen-bonded sheets. The coordination pyramids point upwards along $+\mathbf{b}$ and downwards along $-\mathbf{b}$. The orthorhombic stacking can be converted into the monoclinic one (and vice versa) by moving one layer over $\frac{1}{2} \mathbf{a}$. 

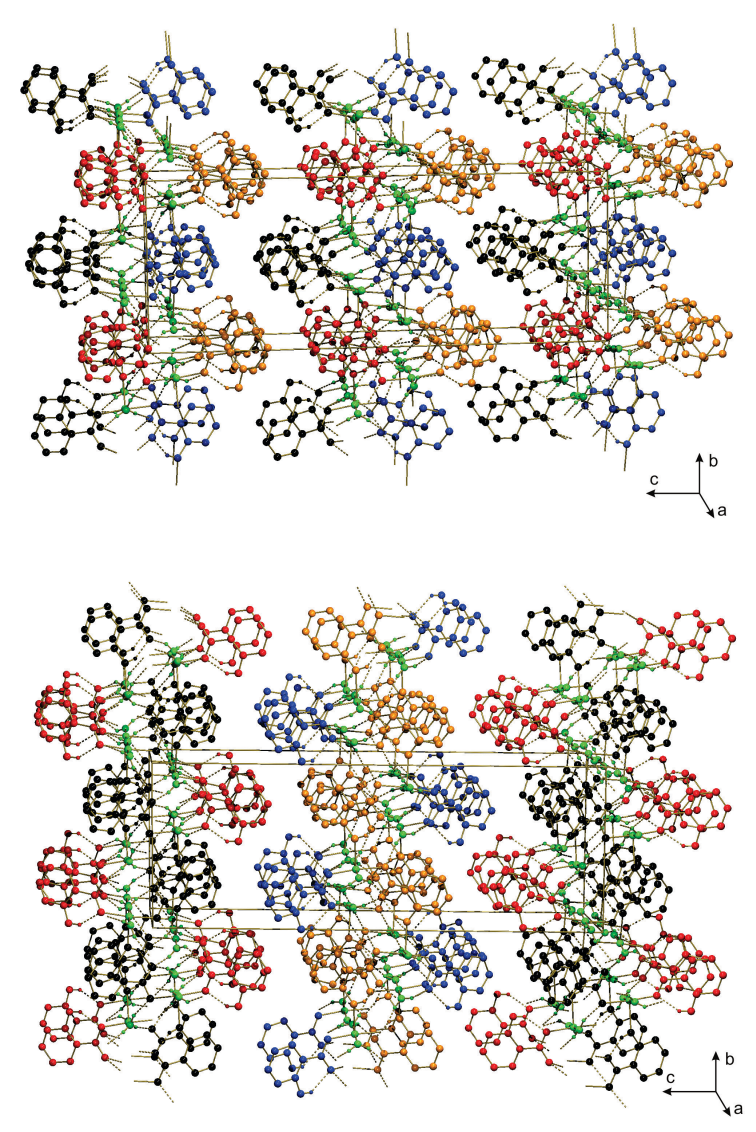

Figure 5. Packing of the hydrogen-bonded layers, viewed approximately along the a axis. $\mathrm{C}-\mathrm{H}$ hydrogen atoms are omitted for clarity. Symmetry-independent salicylate ligands are shown in different colours. The orthorhombic description is shown on top, the monoclinic in the bottom.

For the final results, both structure descriptions have been refined with reflections with $h=$ even only. This makes structure refinement difficult. Convergence of the refinement is slow and can only be achieved with strong restraints. The obtained atomic displacement parameters and the geometric values for distances and angles remain rather unreliable. Similar difficulties with OD structures are described in the literature. ${ }^{[8]}$ Still, the positions of ortho hydroxy groups and of the protonation sites could be reliably determined in the present case. We consider this an important improvement with respect to the earlier descriptions of this structure in the literature.

More information can be obtained from the diffuse streaks with $h=$ odd. This will be considered in the next section.

\section{Stacking Disorder}

The diffusely scattering structure of CuSal can either be described as orthorhombic $P c a 2_{1}$ or monoclinic $P 2_{1} / a$, both
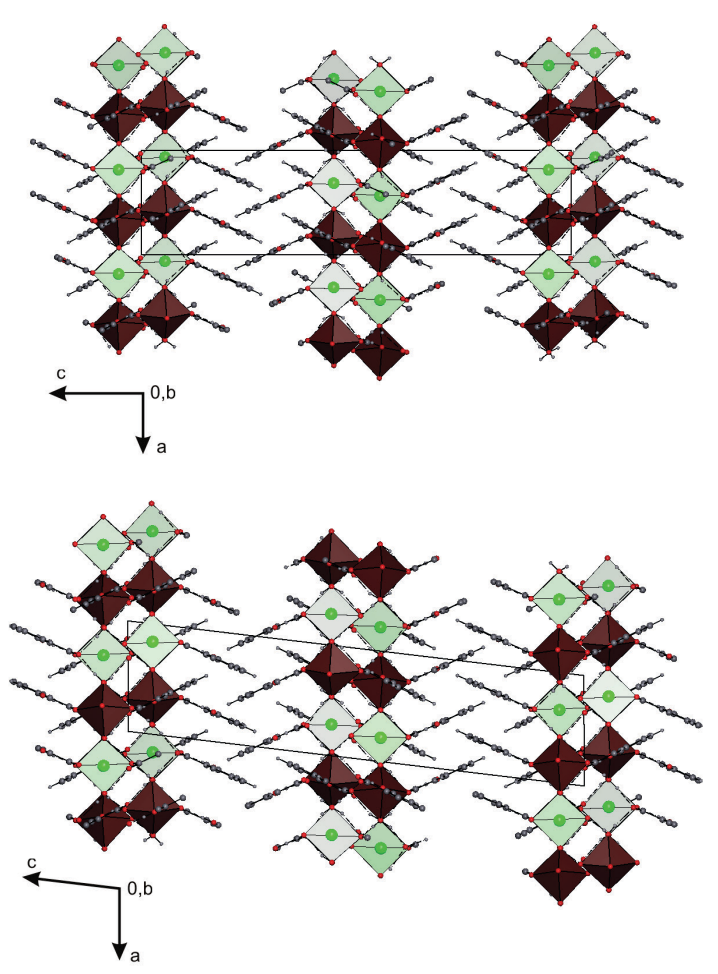

Figure 6. Packing of the hydrogen-bonded layers, viewed along the $\mathbf{b}$ axis. The orthorhombic description is shown on top, the monoclinic on bottom. $\mathrm{CuO}_{5}$ coordination units are drawn as square pyramids. Pyramids pointing in the direction of $+b$ are shown in brown and pointing along $-b$ in green.

with stacking faults along the $\mathbf{c}$ axis. In either case the structure consists of mirror related pairs of ab layers connected by weak van-der-Waals interactions. The stacking fault is a displacement of such a pair over $\frac{1}{2} \mathbf{a}$, which changes the local packing from orthorhombic to monoclinic, or vice versa (Figure 8). A similar one-dimensional disorder has been described for Wollastonite. ${ }^{[17,18]}$ Figure 8 shows the stacking lattice that as such was introduced by Dornberger-Schiff. ${ }^{[19]}$ It has half the size in the a direction. On this lattice the structure is completely ordered. Thus reflections with $h=$ even with respect the original lattice, represent an ordered structure, and can be used to solve the orthorhombic or monoclinic structure. Reflections with $h=$ odd show the loss of long range order in c. In fact reflections $(h=2 n+1, k, l)$ are rods along / (Figure 1 ); these can be interpreted as the Fourier transforms of the arbitrarily stacked ab layers. A mathematical description of scattered intensities follows below.

The structure factor of one unit cell is given by:

$$
F_{A}(h k l)=\sum_{j} f_{j} e^{2 \pi i\left(h x_{j}+k y_{j}+l z_{j}\right)}
$$




$$
F_{C}(h k l)=\sum_{n_{1}} \sum_{n_{2}} \sum_{n_{3}} F_{A}(h k l) e^{\pi i h n_{n_{3}}} e^{2 \pi i\left(n_{1} h+n_{2} k+n_{3} l\right)}
$$

where $p_{n 3}$ in the phase factor is 0 or 1 for the normal and displaced structure factors, respectively. Since the structure is ordered in $\mathbf{a}$ and $\mathbf{b}$ but disordered along $\mathbf{c}$, only integer value of $h$ and $k$ have non-zero contributions, while for $I$ also fractional values will contribute and the resulting structure factor is a continuous function in $l$ :

$$
F_{C}(h k l)=N_{1} N_{2} \sum_{n_{3}} F_{A}(h k l) e^{\pi i h p_{n_{3}}} e^{2 \pi i\left(n_{3} l\right)} .
$$

In case $h=2 n, \mathrm{e}^{\pi i h p n 3}=1$, irrespective of the value $p_{n 3}$ and $F_{C}(\mathrm{hkl})=N_{1} N_{2} N_{3} F_{A}(h k l)$ as for an ordered crystal.

In case $h=2 n+1, \mathrm{e}^{\pi i h p_{n 3}}=\cos \pi h p_{n 3}=S_{n 3}=1$ or -1 for $p_{n 3}=0$ and 1 , respectively, so that we can write for the $h=$ odd reflections:

$$
\begin{aligned}
F_{C}(h k l) & =N_{1} N_{2} \sum_{n_{3}} F_{A}(h k l) S_{n_{3}} e^{2 \pi i\left(n_{3} l\right)} \\
& =N_{1} N_{2} F_{A}(h k l) \sum_{n_{3}} S_{n_{3}} e^{2 \pi i\left(n_{3} l\right)}
\end{aligned}
$$

so it is merely the product of the structure factor $F_{A}(h k l)$ and a lattice summation in / over the $S_{n_{3}}$ values. We performed simulations of the last part of Equation (6), by generating distributions of $S_{n 3}$ or by using correlation coefficients that describe the stacking probability.

\section{Model 1}

along the $\mathrm{c}$ axis. $\mathrm{CuO}_{5}$ coordination units are drawn as square pyramids. Pyramids at height $z \approx 0$ are shown in brown, at height $z \approx 1 / 2$ in green. The orthorhombic description is shown on top, the monoclinic on bottom.

while that of the unit cell displaced over $\frac{1}{2} \mathbf{a}$ is

$$
F_{B}(h k l)=\sum_{j} f_{j} e^{2 \pi i\left(h\left(x_{j}+\frac{1}{2}\right)+k y_{y}+z_{j}\right)}=F_{A} e^{\pi i h} .
$$

The structure factor amplitude for the whole crystal is a lattice summation over the $N_{1} \times N_{2} \times N_{3}$ unit cells:

The first approach is to generate a random sequence of $A$ and $B$ double layers, which is equivalent to selecting random values of $S_{n 3}$, either -1 or 1 . We used a maximum value for $n_{3}=40$ for the crystal size (assuming that no correlations longer than 40 layers occur; this is obviously the case for a random arrangement) and repeated the simulation 2000 times adding the resulting structure factors $F_{c}(h k l)$ incoherently, i.e. the intensity

$$
I(h k l)=\sum_{2000} F_{c}(h k l) \cdot F_{c}^{*}(h k l) .
$$

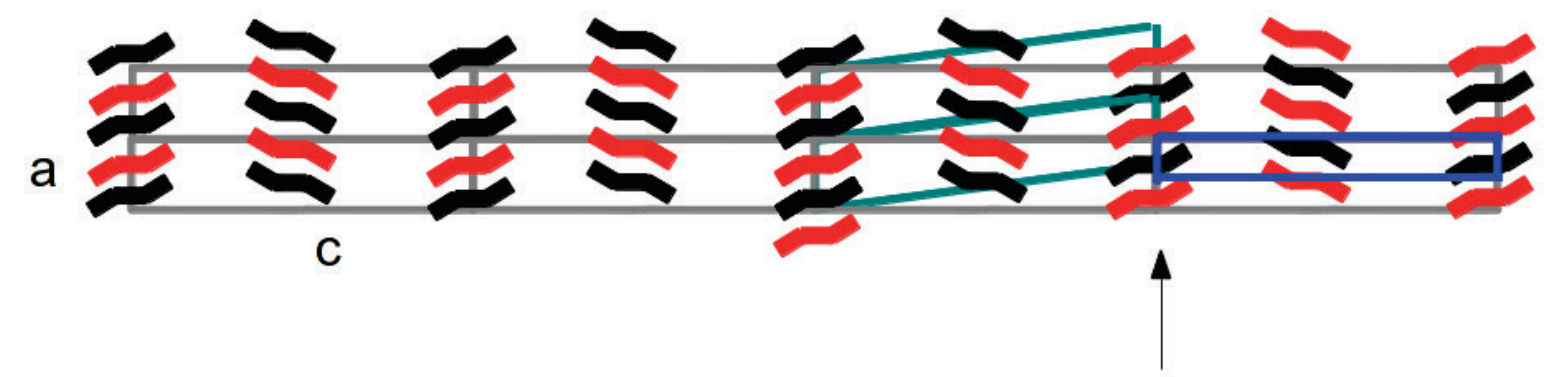

Figure 8. Schematic view of the CuSal structure down the $\boldsymbol{b}$ axis. Orthorhombic unit cell is indicated by grey lines. Layers consist of black and red complexes along a, that are oriented in up and down directions along b, respectively, as seen in Figure 6. A stacking fault of $\frac{1}{2} \mathbf{a}$ is indicated by the arrow, and the molecules pack into the monoclinic lattice, indicated by green lines. The stacking lattice on which all black complexes scatter in phase is indicated by blue lines. 
a)

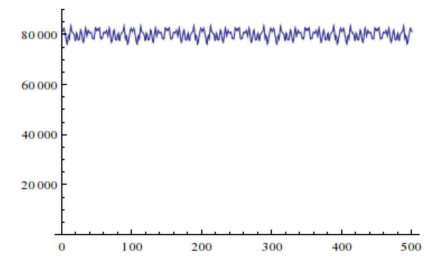

b)

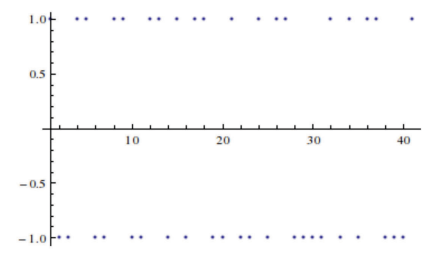

c)

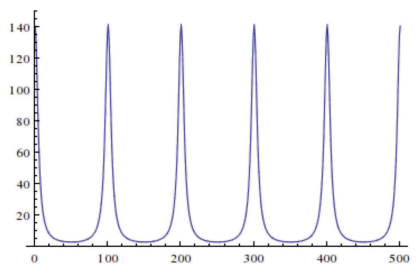

d)

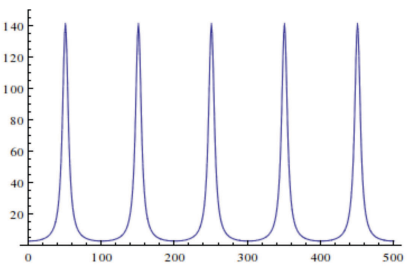

e)

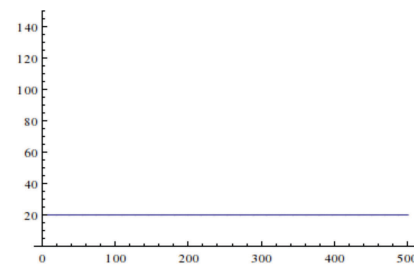

Figure 9. Simulations of the lattice function of Equation (6) for / ranging from 0-5. Model 1: a) Resulting lattice sum as function of I ( $\times 100)$. b) Random sequence of signs $S_{n 3}$ for one crystal of $n_{3}=40$. Model 2: Lattice sums as function of / for the Markov chains: c) Low probability of stacking fault $\alpha=0.1, C_{1}=0.8$, d) larger probability of stacking faults $\alpha=0.9, C_{1}=-0.8$ and e) no preferred nearest neighbour interaction $\alpha=0.5, C_{1}=0$. Only random stacking of double-layers produces rods in reciprocal space.

(hOl)

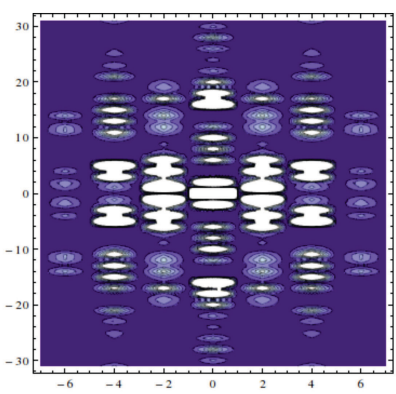

(hOl)

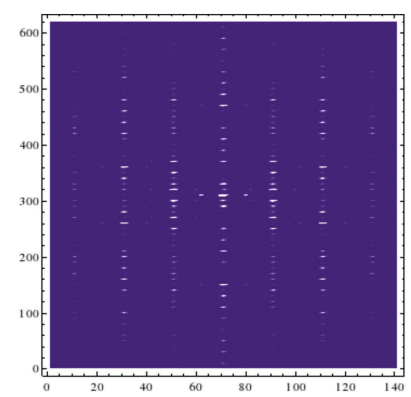

(h1l)

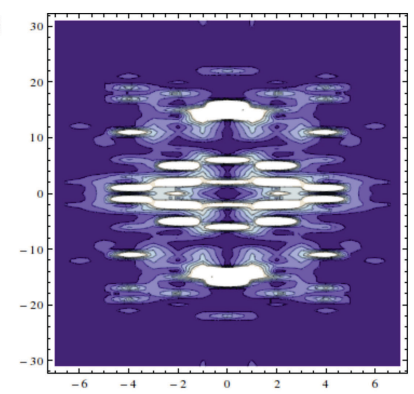

(h1l)

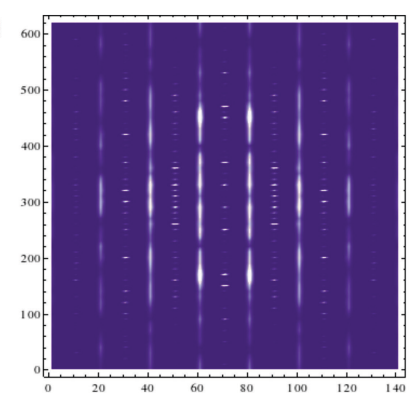

$(1 \mathrm{kl})$

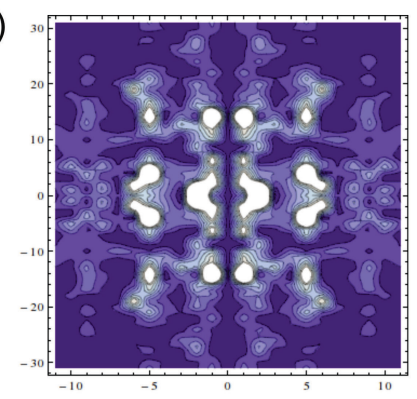

$(1 \mathrm{kl})$

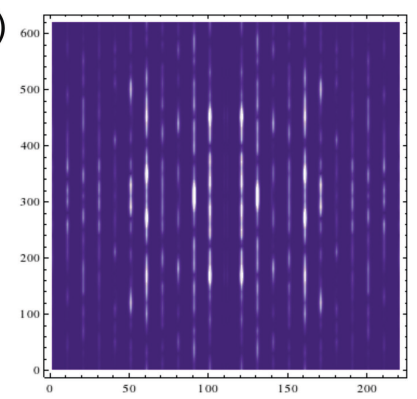

Figure 10. Sections through reciprocal space. Top: unit cell $F_{\text {calc }}^{2}$ of the orthorhombic structure. Bottom: after multiplication with the lattice function.

\section{Model 2}

The correlation of layer stacking can be written in a way similar to Ref. [20] The scattered intensity is

$$
\begin{aligned}
F_{c}(h k l) F_{c}^{*}(h k l) & =N_{1}^{2} N_{2}^{2} \sum_{n_{3}} \sum_{n_{3^{\prime}}} F_{A}(h k l) F_{A}^{*}(h k l) e^{\pi i h\left(p_{n_{3}}-p_{n^{\prime}}\right)} e^{2 \pi i\left(\left(n_{3}-n_{3^{3}}\right) l\right)} \\
& =N_{1}^{2} N_{2}^{2} \sum_{n_{3}} \sum_{n_{3^{\prime}}} F_{A}(h k l) F_{A}^{*}(h k l) S_{n_{3}} S_{n_{3^{\prime}}} e^{2 \pi i\left(\left(n_{3}-n_{3^{3}}\right) l\right)}
\end{aligned}
$$

For $h=$ even all $S_{n 3}$ and $S_{n 3^{\prime}}$ are equal to 1 , and for $h=$ odd Equation (6) can be written as

$$
=N_{1}^{2} N_{2}^{2} F_{A}(h k l) F_{A}^{*}(h k l)\left[\sum_{n=1}^{N_{3}} 2\left(N_{3}-n\right) C_{n} \cos (2 \pi n l)+N_{3}\right]
$$

where the sign function $S_{n_{3}} S_{n^{\prime}}$ is represented by a correlation coefficient $C_{n}$, and $n=n_{3}-n_{3^{\prime}}$. This model for introducing correlation is the so-called Markov chain. Only nearest-neighbour interactions between layers are 

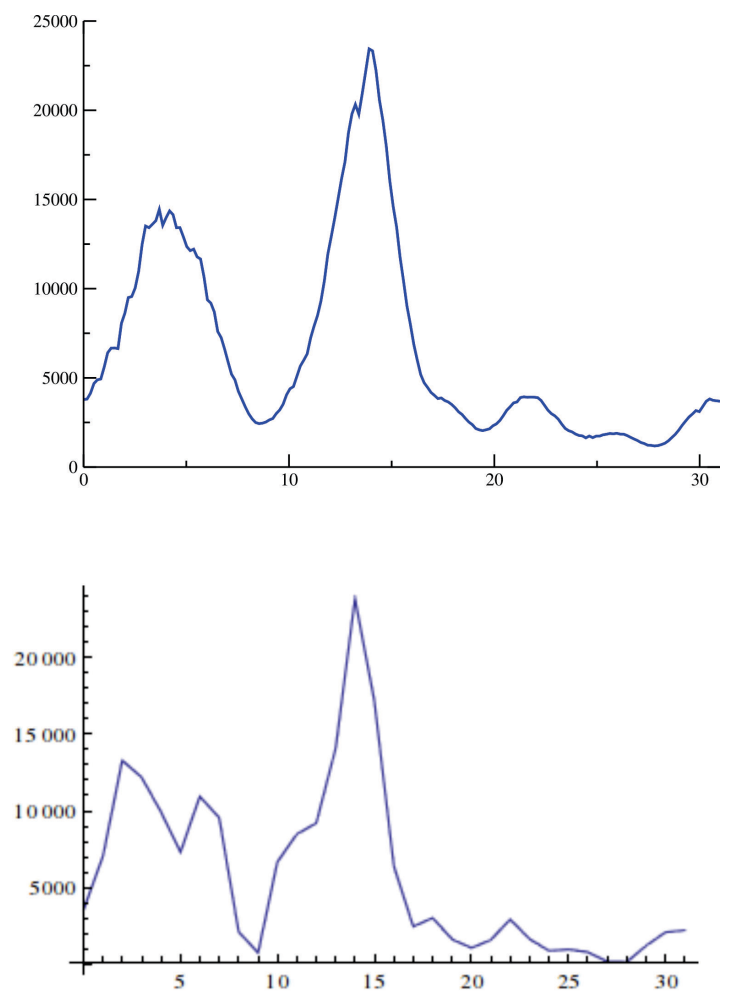

Figure 11. Profile of the $11 /$ direction. The / index is shown on the abscissa. The ordinate shows the intensities on an arbitrary scale. Top: Experimental data from a 3-dimensional reconstruction using the program img $2 \mathrm{hkl}$ of the Eval15 suite. ${ }^{[10]}$ Some instrumental broadening from the diffraction experiment is clearly present. Bottom: Simulation results.

significant and the probability that layer $A$ is stacked on the previous layer is given by $\alpha+C_{1}$ or $\alpha$, for the previous layer being $A$ and $B$, respectively. $\alpha$ is the probability of a stacking fault and $C_{1}$ is the nearest neighbour correlation. Subsequent layers have correlation coefficients $C_{n}=C_{1}^{n}$ as shown by Welberry. ${ }^{[20]}$

\section{Results from the Diffuse Scattering}

The lattice sum of Equation (6), for randomly stacked double-layers (Model 1 ) for $h=2 n+1$ is a nearly constant function in I (Figure 9a). A typical example of the $S_{n_{3}}$ values in a crystallite of 40 unit cells is also shown (Figure 9b). The Markov chain model (Model 2) only gives a constant lattice sum in / for $C_{1}=0$, i.e. no correlation between neighbouring layers; positive $C_{1}$ gives maxima at Bragg positions and negative $C_{1}$ maxima in between those (Figure 9c-e). Thus the diffuse streaks in $c^{*}$ without Bragg spots are only explained when no correlation between stacked layers ( $A$ or $B$ ) exists.
The rods we observe in Figure 1 do not show any Bragg peaks, but also do not have constant intensity. This modulation in intensity ${ }^{[4]}$ is caused by the structure factor $F_{A}(h k l)$ as seen in Equation (6). This can be shown by calculating the continuous unit cell Fourier transform $F_{A}(h k l)$ from the refined orthorhombic structure and then multiplying these with the lattice functions of the ordered lattice $(h=2 n, k, l)$ or the rods $(h=2 n+1, k, l)$. The reconstruction of the experimental data with the program precession (Figure 1) delivers apparent non-integer values due to instrumental broadening. To mimic this in our model calculations, a widened lattice function was applied. The resemblance of the experimental data in Figure 1 with the simulation in Figure 10 is overall very good. As an example, we show the profile along the diffuse $11 /$ direction in Figure 11.

\section{CONCLUSIONS}

We re-determined the crystal structure of CuSal, $\mathrm{Cu}\left(\mathrm{C}_{7} \mathrm{H}_{5} \mathrm{O}_{3}\right)_{2}\left(\mathrm{H}_{2} \mathrm{O}\right)_{2}$, in both the orthorhombic $\left(\mathrm{MDO}_{1}\right)$ and monoclinic phases $\left(\mathrm{MDO}_{2}\right)$. The structure is characterized by one-dimensional coordination polymers in $\mathbf{b}$ direction which are linked by hydrogen bonds into two-dimensional sheets in the ab plane. The crystal diffracts with diffuse streaks along c* for all $h=$ odd, which are caused by packing faults of ordered two-dimensional ab-layers shifted over $\frac{1}{2} \mathbf{a}$, which converts one phases to the other. The $\mathrm{MDO}_{1}$ and $\mathrm{MDO}_{2}$ lattices overlap every second period along $\mathrm{c}$ such that all reflections with $h=$ even are from a ordered structure, be it orthorhombic or monoclinic. Both structures could be solved with $h=$ even reflections only and the hydrogen bonding scheme could be unambiguously established. Diffuse streaks could be explained by a Markow chain growth model similar to Ref. [4]. In contrast to their crystals, our crystal shows complete absence of Bragg reflections on the diffuse streaks, implying random stacking of ab-layers, as we show by lattice sum simulations. Intensity modulation on the diffuse streaks is completely explained by the underlying unit cell Fourier transform of the orthorhombic description. As a consequence of the $\frac{1}{2}$ a shift, which converts one phase to the other, it is irrelevant from which MDO structure we derive our description.

\section{EXPERIMENTAL}

\section{Crystallization}

Green crystals of the title compound were obtained in the same crystallization experiment as the brown crystals of Ref. [6]. 


\section{X-ray Crystal Structure Determination}

A green crystal of approximately $0.06 \times 0.18 \times 0.43 \mathrm{~mm}^{3}$ was mounted on a Bruker Kappa Apexll diffractometer with sealed tube and Triumph monochromator $(\lambda=0.71073 \AA)$ at a temperature of $105(2)$ K. $13 \phi$ and $\omega$ scans were performed with a rotation increment of $0.3^{\circ}$, a detector distance of $60 \mathrm{~mm}$, and an exposure time of $10 \mathrm{~s} /$ frame. Intensity data were integrated with the Eval15 software ${ }^{[10]}$ up to a resolution of $(\sin \theta / \lambda)_{\max }=0.70 \AA^{-1}$. Two integration runs were performed, one with a single orthorhombic lattice (i.e. reciprocal axes matrix) and one with a single monoclinic lattice (i.e. reciprocal axes matrix). The prediction of reflection profiles was based on an isotropic mosaicity of $0.3^{\circ}$ and a mica contribution of 0.5 along $c^{*}$. Absorption correction and scaling of the integrated data was performed with SADABS.[21] This data reduction resulted in two datasets with orthorhombic and monoclinic symmetry, respectively.

Before the structure refinement, the datasets were "de-twinned": the orthorhombic dataset was deprived from the monoclinic contribution and vice versa.

Table 3. Experimental details for the orthorhombic setting. Refined as an inversion twin. Contributions of the monoclinic structure removed from the structure factors

\begin{tabular}{|c|c|c|}
\hline Molecular formula & $\mathrm{Cu}\left(\mathrm{C}_{7} \mathrm{H}_{5} \mathrm{O}_{3}\right)_{2}\left(\mathrm{H}_{2} \mathrm{O}\right)_{2}$ & \\
\hline Formula weight & 373.79 & \\
\hline Crystal system & Orthorhombic & \\
\hline Space group & $\mathrm{Pca}_{1}$ & \\
\hline$a / \AA$ & $7.65385(11)$ & \\
\hline$b / \AA$ & $11.7378(3)$ & \\
\hline$c / \AA$ & $31.5707(6)$ & \\
\hline$V / \AA^{3}$ & $2836.28(9)$ & \\
\hline$Z$ & 8 & \\
\hline$D_{x} / \mathrm{g} \mathrm{cm}^{-3}$ & 1.751 & \\
\hline$\mu / \mathrm{mm}^{-1}$ & 1.58 & \\
\hline refl. condition & $h=$ even, odd & $h=$ even \\
\hline No. of meas. refl. & 82594 & 41161 \\
\hline No. of unique refl. & 8286 & 4342 \\
\hline$R_{\text {int }}$ & 0.0500 & 0.0378 \\
\hline Completeness & $99.9 \%$ & $52.9 \%$ \\
\hline No. of parameters & 420 & 420 \\
\hline No. of restraints & 791 & 791 \\
\hline Twin fraction (BASF) & $0.46(3)$ & $0.50(4)$ \\
\hline R1/wR2 (obs.) & 0.0564 / 0.1589 & $0.0371 / 0.0958$ \\
\hline R1/wR2 (all refl.) & 0.0578 / 0.1609 & $0.0392 / 0.0983$ \\
\hline Goodness of Fit & 1.135 & 1.146 \\
\hline$\Delta \rho_{\max } / \mathrm{e} \AA^{-3}$ & 2.32 & 0.44 \\
\hline$\Delta \rho_{\min } / \mathrm{e} \AA^{-3}$ & -2.66 & -0.53 \\
\hline
\end{tabular}

Reflections with $h=$ even were considered as orthorhombic/monoclinic overlaps, and reflections with $h=$ odd as non-overlapping. The "de-twinning" was based on calculated structure factors. Reflections with negative intensity were not "de-twinned" but omitted. For the calculation of the monoclinic $F_{\text {calc }}^{2}$ a rotation twin about $c^{*}$ was assumed with a twin fraction of $50 \%$. The calculation of the orthorhombic $F_{\text {calc }}^{2}$ was based on a $50 \%$ inversion twin. In the case of the orthorhombic dataset, the $F_{\text {calc }}^{2}$ were scaled with FVAR $=0.21$ for the orthorhombic, and FVAR = 0.18 for the monoclinic contribution. For the monoclinic dataset, the corresponding scale factors were FVAR $=0.21$ for the monoclinic, and FVAR $=0.17$ for the orthorhombic contribution. This slight inconsistency in scale factors is probably a consequence of the diffuse intensities in the $h=$ odd layers.

Coordinates for the starting models were taken from Ref. [2]. The orientational disorder of the salicylate molecules could not be reproduced and we refined ordered salicylate ligands with the hydroxy group on only one of the ortho positions on the aromatic ring.

Table 4. Experimental details for the monoclinic setting. Refined as a rotation twin. Contributions of the orthorhombic structure removed from the structure factors.

\begin{tabular}{|c|c|c|}
\hline Molecular formula & $\mathrm{Cu}\left(\mathrm{C}_{7} \mathrm{H}_{5} \mathrm{O}_{3}\right)_{2}\left(\mathrm{H}_{2} \mathrm{O}\right)_{2}$ & \\
\hline Formula weight & 373.79 & \\
\hline Crystal system & Monoclinic & \\
\hline Space group & $P 2_{1} / a$ & \\
\hline$a / \AA$ & $7.6558(2)$ & \\
\hline$b / \AA$ & $11.7408(3)$ & \\
\hline$c / \AA$ & $31.8085(12)$ & \\
\hline$\beta /^{\circ}$ & $96.931(2)$ & \\
\hline$V / \AA^{3}$ & $2838.22(15)$ & \\
\hline$Z$ & 8 & \\
\hline$D_{x} / \mathrm{g} \mathrm{cm}^{-3}$ & 1.750 & \\
\hline$\mu / \mathrm{mm}^{-1}$ & 1.58 & \\
\hline refl. condition & $h=$ even, odd & $h=$ even \\
\hline No. of meas. refl. & 86764 & 43435 \\
\hline No. of unique refl. & 8262 & 4322 \\
\hline$R_{\text {int }}$ & 0.0508 & 0.0390 \\
\hline Completeness & $99.7 \%$ & $52.2 \%$ \\
\hline No. of parameters & 420 & 420 \\
\hline No. of restraints & 774 & 774 \\
\hline Twin fraction (BASF) & $0.4627(18)$ & $0.501(6)$ \\
\hline R1/wR2 (obs.) & $0.0650 / 0.1845$ & $0.0431 / 0.1107$ \\
\hline R1/wR2 (all refl.) & 0.0678 / 0.1876 & $0.0473 / 0.1132$ \\
\hline Goodness of Fit & 1.064 & 1.212 \\
\hline$\Delta \rho_{\max } / \mathrm{e} \AA^{-3}$ & 3.21 & 0.40 \\
\hline$\Delta \rho_{\min } /$ e $\AA^{-3}$ & -2.02 & -0.55 \\
\hline
\end{tabular}


Least-squares refinement was performed with SHELXL-2018. ${ }^{[22]}$ A rather large number of restraints was used in the refinement concerning the geometric equivalence between the salicylate ligands and their flatness (instructions SAME and FLAT). Non-hydrogen atoms were refined with anisotropic displacement parameters and restrained using $I S O R$ and RIGU instructions. Hydrogen atoms of the water molecules were kept fixed on their positions from difference-Fourier maps. All other hydrogen atoms were refined with a riding model. The orthorhombic structure was refined as inversion twin. For the monoclinic structure refinement we used a twin matrix of $(-1,0,0 /$ $0,-1,0 / 1,0,1)$ which corresponds to a twofold rotation about $h k l=(0,0,1)$ or $u v w=[-1,0,-2]$.

Summaries of the structural details are given in Table 3 and Table 4. The Tables give the results for refinements on all data and on data with $h=$ even only. Figures of the molecular structures were prepared with the programs PLATON ${ }^{[16]}$ and ToposPro. ${ }^{[23]}$ CCDC 1841406-1841408 and 1850913 contain the supplementary crystallographic data for this paper. These data can be obtained free of charge from The Cambridge Crystallographic Data Centre via www.ccdc.cam.ac.uk/data_request/cif.

\section{Simulations}

Simulations of lattice sum of Equation (7) are carried out with Mathematica. ${ }^{[24]}$ The summation is over 40 unit cells in the c direction $\left(n_{3}=40\right)$ and repeated 2000 times. The structure factor $F_{A}(h k l)$ is set to 1.0 , and also $N_{1}=N_{2}=1$. For model 1 , random values of -1 or 1 were generated for $p_{n 3}$ using the RandomInteger function in Mathematica. For model 2, Equation (8) was calculated, while setting $N_{1}=N_{2}$ $=1$ and $F_{A}=F_{A}^{*}=1 . C_{1}$ is the nearest neighbour correlation and $\alpha$ is the probability of a stacking fault. The two parameters are related through $\alpha=m_{A}\left(1-C_{1}\right)$, where $m_{A}$ is the fraction of layers $A$ in the crystal, which we assume to be 0.5 . The probability of stacking the same layers ( $A$ on $A$ and $B$ on $B$ ) is $\alpha+C_{1}$, while that of stacking different layers ( $A$ on $B$ or $B$ on $A$ ) is $\alpha$. Inclusion of values for $F_{A}(h k l)$ in Equation (6) leads to modulation of the diffuse scattering on the rods with $h=2 n+1$. $F_{A}^{2}$ values were calculated from the coordinates of the orthorhombic structure (setting $B=$ $2.0 \AA^{2}$ ) at integer values of $h k l$; intermediate values are obtained through interpolation with Mathematica Interpolation (Figure 10 (top)). As the simulations have shown that only Bragg spots or diffuse lines occur, we represented the lattice sums of Equation (6) by a lattice function having sharp maxima at Bragg positions and rods for the diffuse layer lines; these are multiplied with $F_{A}^{2}$ to get the images in Figure 10 (bottom).

\section{REFERENCES}

[1] K. Dornberger-Schiff, T. I. Malinowski, Acta Crystallogr. 1966, 21, A171.

[2] S. Jagner, R. G. Hazell, K. P. Larsen, Acta Crystallogr., Sect. B: Struct. Crystallogr. Cryst. Chem. 1976, 32, 548.

[3] S. Tjotta, E. J. Samuelsen, S. Jagner, J. Phys.: Condens. Matter 1991, 3(20), 3411.

[4] E. J. Samuelsen, S. Tjotta, S. Jagner, J. Phys.: Condens. Matter 1991, 3(20), 3421.

[5] E. J. Samuelsen, S. Jagner, Phase Transitions 1993, 43(1-4), 89.

[6] J. A. v. d. Horn, M. Lutz, Acta Crystallogr., Sect. E: Struct. Rep. Online 2017, 73, 235.

[7] G. Ferraris, E. Makovicky, and S. Merlino, Crystallography of Modular Materials, vol. 15 of IUCr Monographs of Crystallography, ch. 2, pp. 127-206. Oxford University Press, 2004.

[8] S. Ďurovič, J. Hybler, Z. Kristallogr. - Cryst. Mater. 2006, 221(1), 63.

[9] B. Stöger, Symmetry 2014, 6(3), 589.

[10] A. M. M. Schreurs, X. Xian, L. M. J. Kroon-Batenburg, J. Appl. Crystallogr. 2010, 43, 70.

[11] T. L. Falch, J. B. Fløystad, D. W. Breiby, A. C. Elster, IEEE Access 2013, 1, 564.

[12] J. A. van der Horn, B. Souvignier, M. Lutz, Crystals 2017, 7(12), 377.

[13] A. W. Addison, T. N. Rao, J. Reedijk, J. van Rijn, G. C. Verschoor, J. Chem. Soc., Dalton Trans. 1984, 1349.

[14] M. Lutz, A. L. Spek, Acta Crystallogr., Sect. C: Cryst. Struct. Commun. 2009, 65, m69.

[15] H. D. Flack, Acta Crystallogr., Sect. A: Found. Crystallogr. 1983, 39, 876.

[16] A. L. Spek, Acta Crystallogr., Sect. D: Biol. Crystallogr. 2009, 65, 148.

[17] B. T. M. Willis, Proc. R. Soc. Lond. A 1958, 248(1253), 183.

[18] W. A. Wooster, Diffuse X-Ray reflections from crystals. New York: Dover, 1997.

[19] K. Dornberger-Schiff, Acta Crystallogr. 1956, 9, 593.

[20] T. R. Welberry, Diffuse X-ray Scattering and Models of Disorder, vol. 16 of $\mathrm{IUCr}$ Monographs on Crystallography. Oxford University Press, 2004.

[21] L. Krause, R. Herbst-Irmer, G. M. Sheldrick, D. Stalke, J. Appl. Crystallogr. 2015, 48, 3.

[22] G. M. Sheldrick, Acta Crystallogr., Sect. C: Struct. Chem. 2015, 71, 3.

[23] V. A. Blatov, A. P. Shevchenko, D. M. Proserpio, Cryst. Growth Des. 2014, 14(7), 3576.

[24] Wolfram Research Inc., Mathematica 9.0, 2012. 